\title{
Bayesian Estimation and Prediction for the Power Law Process with Left-Truncated Data
}

\author{
Guo-Liang Tian ${ }^{1}$, Man-Lai Tang ${ }^{2}$ and Jun-Wu Yu ${ }^{3}$ \\ ${ }^{1}$ The University of Hong Kong, ${ }^{2}$ Hong Kong Baptist University \\ and ${ }^{3}$ Hunan University of Science and Technology
}

\begin{abstract}
The power law process (PLP) (i.e., the nonhomogeneous Poisson process with power intensity law) is perhaps the most widely used model for analyzing failure data from reliability growth studies. Statistical inferences and prediction analyses for the PLP with left-truncated data with classical methods were extensively studied by $\mathrm{Yu}$ et al. (2008) recently. However, the topics discussed in Yu et al. (2008) only included maximum likelihood estimates and confidence intervals for parameters of interest, hypothesis testing and goodness-of-fit test. In addition, the prediction limits of future failure times for failure-truncated case were also discussed. In this paper, with Bayesian method we consider seven totally different prediciton issues besides point estimates and prediction limits for $x_{n+k}$. Specifically, we develop estimation and prediction methods for the PLP in the presence of left-truncated data by using the Bayesian method. Bayesian point and credible interval estimates for the parameters of interest are derived. We show how five single-sample and three two-sample issues are addressed by the proposed Bayesian method. Two real examples from an engine development program and a repairable system are used to illustrate the proposed methodologies.
\end{abstract}

Key words: Bayesian method, nonhomogeneous Poisson process, noninformative prior, prediction intervals, reliability growth.

\section{Introduction}

When failure times from different systems during their development programs are collected and analyzed, an approximate straight line pattern in the corresponding log-log plot of the cumulative mean time between failures (MTBF) against the cumulative operating time is usually observed (see, Duane, 1964). Crow (1974) extended the Duane model to the nonhomogeneous Poisson process (NHPP) with a power intensity law, which is also known as AMSAA model due to its adoption by the U.S. Army Materiel Systems Analysis Activity. The 
NHPP model is generally used to monitor the reliability growth of repairable systems, to assess the reliability growth of software and to predict failure behaviors. Statistically, a NHPP $\{N(t), t \geq 0\}$ with the power intensity law

$$
\lambda(t)=(\beta / \alpha)(t / \alpha)^{\beta-1}, \quad \alpha, \beta>0,
$$

is also known as power law process (PLP) or Weibull process. The corresponding mean function is defined by

$$
m(t)=E\{N(t)\}=\int_{0}^{t} \lambda(s) d s=(t / \alpha)^{\beta} .
$$

It was shown by Rigdon (2002) that a linear Duane plot does not imply a PLP, and a PLP does not imply a linear Duane plot.

Assume that we conduct a reliability growth test on some repairable system in the time interval $(0, t]$. For the failure truncated case, the number of failures, $n$, is predetermined. Let $0<x_{1}<x_{2}<\cdots<x_{n}$ be the first $n$ ordered failure times of the PLP. The time to the first failure (i.e., $x_{1}$ ) can be shown to follow Weibull distribution with scale parameter $\alpha$ and shape parameter $\beta$. That is, the probability density function (pdf) of $x_{1}$ is (Crowder et al., 1991)

$$
f_{1}\left(x_{1}\right)=\frac{\beta}{\alpha}\left(\frac{x_{1}}{\alpha}\right)^{\beta-1} e^{-\left(x_{1} / \alpha\right)^{\beta}}, \quad x_{1}>0
$$

with the corresponding distribution function

$$
F_{1}\left(x_{1}\right)=1-e^{-\left(x_{1} / \alpha\right)^{\beta}}, \quad x_{1}>0 .
$$

Let $f_{i}\left(x_{i} \mid x_{1}, \cdots, x_{i-1}\right)$ denote the conditional density function of $x_{i}$ given $x_{1}, \cdots$, $x_{i-1}$, we have

$$
f_{i}\left(x_{i} \mid x_{1}, \cdots, x_{i-1}\right)=\frac{\beta}{\alpha}\left(\frac{x_{i}}{\alpha}\right)^{\beta-1} \exp \left[-\left(\frac{x_{i}}{\alpha}\right)^{\beta}+\left(\frac{x_{i-1}}{\alpha}\right)^{\beta}\right], \quad x_{i}>x_{i-1} .
$$

The joint pdf of $x_{1}, \cdots, x_{n}$ is thus given by

$$
\begin{aligned}
f\left(x_{1}, \cdots, x_{n}\right) & =f_{1}\left(x_{1}\right) \prod_{i=2}^{n} f_{i}\left(x_{i} \mid x_{1}, \cdots, x_{i-1}\right) \\
& =\left(\frac{\beta}{\alpha}\right)^{n} e^{-\left(x_{n} / \alpha\right)^{\beta}} \prod_{i=1}^{n}\left(\frac{x_{i}}{\alpha}\right)^{\beta-1}, \quad 0<x_{1}<\cdots<x_{n} .
\end{aligned}
$$


For the time truncated case, the test time $t$ is predetermined. Let $0<x_{1}<\cdots<$ $x_{n}<t$ be the observed failure times of the PLP. Similar to (1.3), the joint pdf of $\left(x_{1}, \cdots, x_{n} ; N=n\right)$ is given by

$$
\begin{aligned}
f\left(x_{1}, \cdots, x_{n} ; N=n\right)= & \left(\frac{\beta}{\alpha}\right)^{n} e^{-(t / \alpha)^{\beta}} \prod_{i=1}^{n}\left(\frac{x_{i}}{\alpha}\right)^{\beta-1}, \\
& 0<x_{1}<\cdots<x_{n}<t, \quad n>0, \\
\operatorname{Pr}\{N=0\}= & e^{-(t / \alpha)^{\beta}}, \quad n=0,
\end{aligned}
$$

where $N \hat{=} N(t)$ and the symbol $\hat{=}$ means "equal by definition".

In practice, left-truncated data occur due to various reasons such as not being able to observe in the early developmental phase of a testing program. Recently, Yu et al. (2008) developed classical methods for statistical inferences and prediction analyses for the PLP with the first $r-1$ failure times (i.e., $\left\{x_{i}\right\}_{i=1}^{r-1}$ ) being missing. Using their notations, we denote the observed data as $Y_{\mathrm{obs}}^{\mathrm{ft}}=\left\{x_{r}, \cdots, x_{n}\right\}$ for the failure-truncated case and $Y_{\mathrm{obs}}^{\mathrm{tt}}=\left\{x_{r}, \cdots, x_{n} ; t\right\}$ for the time-truncated case. First, the topics discussed in Yu et al. (2008) only included maximum likelihood estimates and confidence intervals for parameters of interest (e.g., $\alpha, \beta$ and MTBF), hypothesis testing on $\alpha$ and $\beta$, and goodnessof-fit test. In addition, the prediction limit of the $(n+k)$-th future failure time (i.e., $x_{n+k}$ ) for failure-truncated case was also discussed. In this paper, we will consider seven totally different issues (see, Sections 4.2-4.4 and 5) besides point estimates (see, Section 3) and prediction limits for $x_{n+k}$ (see, Section 4.1), and solutions to the seven prediction issues are not yet available to date for both classical and Bayesian methods. Second, the prediction limit of $x_{n+k}$ was available only for the failure-truncated case (Yu et al., 2008). A similar result does not yet exist for the time-truncated case. The main reason is our inability to find an adequate prediction statistic in the framework of classical methods. Fortunately, the Bayesian method can be applied (see, Section 4.1). Finally, there is a computational challenge in obtaining exact solutions from the classical methods and the accuracy of approximate formulae are heavily dependent of large sample sizes, while the Bayesian method can facilitate the computation by employing the conditional sampling procedure (see, two conditional sampling procedures in Sections 3.1 and 3.3).

This article aims to develop Bayesian estimation and prediction methods for the PLP with the first $r-1$ failure times being missing. This left-truncated data pattern commonly occurs when (i) the importance of a reliability growth program is eventually recognized only when manufacturers reported the failures several times; and (ii) a new data-recording person may not be able to determine the exact failure times during the early stage of the process. This article is organized as follows. Section 2 presents the posterior and predictive distributions. 
Section 3 derives Bayesian point estimates and credible interval estimates for the parameters of interest. Five practical single-sample issues are discussed and then addressed by the Bayesian approach in Section 4. In Section 5, we develop Bayesian methods for three two-sample issues. Two real examples from an engine development program and a repairable system are used to illustrate the proposed methodologies in Section 6. A brief discussion is given in Section 7.

\section{Posterior and Predictive Distributions}

In this article, we consider a common scenario in which missing data are produced only in the early stage of the test time (see, Yu et al., 2008). That is, $\left\{x_{i}\right\}_{i=1}^{r-1}$ are missing. For the failure-truncated PLP, the joint pdf of the observed data $Y_{\mathrm{obs}}^{\mathrm{ft}}=\left\{x_{i}\right\}_{i=r}^{n}$ is

$$
f\left(x_{r}, \cdots, x_{n}\right)=\frac{\beta^{n-r+1} \exp \left[-\left(x_{n} / \alpha\right)^{\beta}\right]}{(r-1) ! \alpha^{n \beta}} \cdot x_{r}^{(r-1) \beta} \prod_{i=r}^{n} x_{i}^{\beta-1}, \quad 0<x_{1}<\cdots<x_{n} .
$$

For the time-truncated PLP, we also assume that $\left\{x_{i}\right\}_{i=1}^{r-1}$ are missing. The joint pdf of the observed data $Y_{\text {obs }}^{\mathrm{tt}}=\left\{x_{r}, \cdots, x_{n} ; t\right\}$ is (Yu et al., 2008)

$$
f\left(x_{r}, \cdots, x_{n} ; N=n\right)=\frac{\beta^{n-r+1} \exp \left[-(t / \alpha)^{\beta}\right]}{(r-1) ! \alpha^{n \beta}} \cdot x_{r}^{(r-1) \beta} \prod_{i=r}^{n} x_{i}^{\beta-1},
$$

where $0<x_{1}<\cdots<x_{n}<t$. Let $Y_{\text {obs }}$ represent $Y_{\text {obs }}^{\mathrm{ft}}$ or $Y_{\text {obs }}^{\mathrm{tt}}$. Combining (2.1) with (2.2), the likelihood function for $\alpha$ and $\beta$ is then given by

$$
L\left(\alpha, \beta \mid Y_{\mathrm{obs}}\right)=[(r-1) !]^{-1} \alpha^{-n \beta} \beta^{n-r+1} \exp \left[-(\tau / \alpha)^{\beta}\right] \cdot x_{r}^{(r-1) \beta} u^{\beta-1}, \quad \alpha, \beta>0,
$$

where

$$
u \hat{=} \prod_{i=r}^{n} x_{i}, \quad \text { and } \quad \tau \hat{=}\left\{\begin{array}{cl}
x_{n}, & \text { if } Y_{\mathrm{obs}}=Y_{\mathrm{obs}}^{\mathrm{ft}} \\
t, & \text { if } Y_{\mathrm{obs}}=Y_{\mathrm{obs}}^{\mathrm{tt}}
\end{array}\right.
$$

When prior information is not available, it is reasonable to use noninformative prior distributions.

Case 1: Shape parameter $\boldsymbol{\beta}$ is known. Following Guida et al. (1989), we choose the following noninformative prior density of $\alpha$

$$
g(\alpha) \propto 1 / \alpha, \quad \alpha>0 .
$$

The posterior distribution of $\alpha$ is thus given by

$$
\begin{aligned}
h\left(\alpha \mid Y_{\text {obs }}\right) & \propto L\left(\alpha, \beta \mid Y_{\text {obs }}\right) \times g(\alpha) \\
& =[\Gamma(n)]^{-1} \beta \tau^{n \beta} \alpha^{-n \beta-1} \exp \left[-(\tau / \alpha)^{\beta}\right], \quad \alpha>0 .
\end{aligned}
$$


Let $y$ be any random variable that one wants to predict (e.g., $x_{n+k}$ with $k$ being any positive integer); the predictive density of $y$ is

$$
f\left(y \mid Y_{\mathrm{obs}}\right)=\int_{0}^{\infty} f\left(y \mid Y_{\mathrm{obs}}, \alpha\right) h\left(\alpha \mid Y_{\mathrm{obs}}\right) d \alpha .
$$

Hence, the Bayesian upper prediction limit (UPL) of $y$ with level $\gamma$, denoted as $y_{U}^{(\beta)}$, satisfies

$$
\gamma=\int_{-\infty}^{y_{U}^{(\beta)}} f\left(y \mid Y_{\mathrm{obs}}\right) d y .
$$

Case 2: Shape parameter $\boldsymbol{\beta}$ is unknown. Following Box \& Tiao (1973), we consider the following noninformative joint prior density for $(\alpha, \beta)$

$$
g(\alpha, \beta) \propto 1 /(\alpha \beta), \quad \alpha, \beta>0 .
$$

Hence, the corresponding joint posterior density is

$$
h\left(\alpha, \beta \mid Y_{\text {obs }}\right)=c^{-1} \beta^{n-r}\left(u x_{r}^{r-1}\right)^{\beta} \cdot \alpha^{-n \beta-1} \exp \left[-(\tau / \alpha)^{\beta}\right], \quad \alpha, \beta>0,
$$

where

$$
c \hat{=} \Gamma(n) \Gamma(n-r) / z^{n-r},
$$

and

$$
z \hat{=} \sum_{i=r+1}^{n} \ln \left(\tau / x_{i}\right)+r \ln \left(\tau / x_{r}\right) .
$$

Similar to (2.7) and (2.8), let $y_{U}$ denote the Bayesian UPL of $y$ with level $\gamma$. Hence, we have

$$
f\left(y \mid Y_{\text {obs }}\right)=\int_{0}^{\infty} \int_{0}^{\infty} f\left(y \mid Y_{\text {obs }}, \alpha, \beta\right) h\left(\alpha, \beta \mid Y_{\text {obs }}\right) d \alpha d \beta,
$$

and

$$
\gamma=\int_{-\infty}^{y_{U}} f\left(y \mid Y_{\text {obs }}\right) d y
$$

\section{Bayesian Point and Credible Interval Estimates}

\subsection{Bayesian estimates of $\alpha$ and $\beta$}

We first consider the case in which $\beta$ is known. Let

$$
X \hat{=}(\tau / \alpha)^{\beta} .
$$


The inverse transformation is then $\alpha=\tau X^{-1 / \beta}$. From (2.6), it is easy to verify that

$$
X \mid Y_{\text {obs }} \sim \Gamma(n, 1) \text { or } 2 X \mid Y_{\text {obs }} \sim \chi^{2}(2 n) .
$$

Therefore, the Bayes point estimator and two-sided $100 \gamma \%$ Bayes credible interval (CI) for $\alpha$ are respectively given by

$$
\tilde{\alpha}=E\left(\alpha \mid Y_{\text {obs }}\right)=\tau E\left(X^{-1 / \beta} \mid Y_{\text {obs }}\right)=\tau \Gamma\left(n-\frac{1}{\beta}\right) / \Gamma(n),
$$

and

$$
\left[\tau\left\{\frac{1}{2} \chi^{2}(2 n ;(1+\gamma) / 2)\right\}^{-1 / \beta}, \tau\left\{\frac{1}{2} \chi^{2}(2 n ;(1-\gamma) / 2)\right\}^{-1 / \beta}\right]
$$

where $\chi^{2}(n ; \gamma)$ denotes the $\gamma$ percentage point of the chi-square distribution with $n$ degrees of freedom such that $\operatorname{Pr}\left\{\chi^{2}(n) \leq \chi^{2}(n ; \gamma)\right\}=\gamma$.

When $\beta$ is unknown, we can integrate (2.10) with respect to $\alpha$ to obtain the following marginal posterior density of $\beta$

$$
h\left(\beta \mid Y_{\mathrm{obs}}\right)=\frac{z^{n-r}}{\Gamma(n-r)} \beta^{n-r-1} e^{-\beta z}, \quad \beta>0 .
$$

Obviously, (3.5) implies that

$$
\beta \mid Y_{\mathrm{obs}} \sim \Gamma(n-r, z) \text { or } 2 z \beta \mid Y_{\mathrm{obs}} \sim \chi^{2}(2 n-2 r) .
$$

Based on (3.6), the Bayes point estimator and two-sided $100 \gamma \%$ Bayes CI for $\beta$ are respectively given by

$$
\tilde{\beta}=(n-r) / z,
$$

and

$$
\left[\chi^{2}(2 n-2 r ;(1-\gamma) / 2) /(2 z), \chi^{2}(2 n-2 r ;(1+\gamma) / 2) /(2 z)\right] .
$$

By comparing (3.8) with (3.11) in Yu, Tian and Tang (2008), we can immediately conclude that the Bayes CI for $\beta$ is identical to the classical confidence interval for $\beta$ when the joint prior is chosen to be (2.9).

To derive the Bayes estimates for $\alpha$, we adopt the conditional sampling method. From (2.10), we have

$$
h\left(\alpha \mid Y_{\text {obs }}, \beta\right) \propto \alpha^{-n \beta-1} \exp \left[-(\tau / \alpha)^{\beta}\right] .
$$

Similar to (3.2), we obtain

$$
X \mid\left(Y_{\text {obs }}, \beta\right) \sim \Gamma(n, 1),
$$

where $X$ is defined in (3.1). Hence, the conditional sampling algorithm can be summarized as follows.

The CONDITIONAL SAMPLING: 
Step 1. Generate $m$ i.i.d. posterior samples $\left\{\beta^{(\ell)}\right\}_{\ell=1}^{m}$ of $\beta$ according to (3.6);

Step 2. Generate $m$ i.i.d. posterior samples $\left\{X^{(\ell)}\right\}_{\ell=1}^{m}$ of $X$ according to (3.9);

Step 3. For each given pair $\left(\beta^{(\ell)}, X^{(\ell)}\right)$, calculate $\alpha^{(\ell)}=\tau\left(X^{(\ell)}\right)^{-1 / \beta^{(\ell)}}$. Here, $\left\{\alpha^{(\ell)}\right\}_{\ell=1}^{m}$ are i.i.d. posterior samples of $\alpha$.

The Bayes estimates for $\alpha$ can then be obtained from the above i.i.d. posterior samples $\left\{\alpha^{(\ell)}\right\}_{\ell=1}^{m}$. For example, the Bayes point estimator of $\alpha$ is then given by $\tilde{\alpha}=\frac{1}{m} \sum_{\ell=1}^{m} \alpha^{(\bar{\ell})}$.

\subsection{The Pseudo Bayesian Estimate of $M\left(x_{n}\right)$}

In this subsection, we restrict our discussion to the failure-truncated case. If no improvements are incorporated into the repairable system after the time of the $n$-th failure (i.e., $\left.x_{n}\right)$ and the intensity $\lambda\left(x_{n}\right)=(\beta / \alpha)\left(x_{n} / \alpha\right)^{\beta-1}$ remains constant thereafter, then the subsequent times between failures of the system independently follow exponential distribution with the common failure rate $\lambda\left(x_{n}\right)$ and the MTBF $M\left(x_{n}\right) \hat{=} 1 / \lambda\left(x_{n}\right)$. Since $M\left(x_{n}\right)$ involves the random variable $x_{n}$, its posterior density cannot be obtained. ${ }^{1}$ Following the idea of Higgins and Tsokos (1981), we consider the pseudo Bayes point and CI estimates for $\lambda\left(x_{n}\right)$ (or $M\left(x_{n}\right)$ equivalently) instead. For this purpose, we noted that (see, Theorem 1 in $\mathrm{Yu}$ et al., 2008)

$$
\frac{\lambda\left(x_{n}\right)}{\hat{\lambda}_{M}\left(x_{n}\right)}=\frac{Z \cdot S}{4 n(n-r+1)},
$$

where $Z \sim \chi^{2}(2 n-2 r)$ is independent of $S \sim \chi^{2}(2 n)$ and $\hat{\lambda}_{M}\left(x_{n}\right)=n(n-r+$ $1) /\left(z x_{n}\right)$ is the maximum likelihood estimate of $\lambda\left(x_{n}\right)$. For the sake of convenience, we define

$$
\lambda_{n} \hat{=} \lambda\left(x_{n}\right), \quad \hat{\lambda}_{M} \hat{=} \hat{\lambda}_{M}\left(x_{n}\right), \quad a \hat{=} \frac{2 n(n-r+1)}{2 n-r+1}, \quad \text { and } \quad Q \hat{=} \frac{a \lambda_{n}}{\hat{\lambda}_{M}} .
$$

As a result, we have

$$
E(Q)=n^{*} \text { and } \operatorname{Var}(Q)=2 n^{*}
$$

where

$$
n^{*}=\frac{2 n(n-r)}{2 n-r+1} .
$$

\footnotetext{
${ }^{1}$ Some researchers think that although the failure time $x_{n}$ is a random variable, it has been observed before making posterior inference. Therefore, the posterior Bayesian estimates of $\lambda\left(x_{n}\right)$ and $M\left(x_{n}\right)$ in a failure-truncated PLP can be easily obtained through the same procedure used for the time-truncated case (see Section 3.3).
} 
By treating $\lambda_{n}$ as a parameter, we consider the transformation $\hat{\lambda}_{M}=a \lambda_{n} / Q$. Since (3.10) implies that $Q$ may be approximated by the chi-square random variable $\chi^{2}\left(n^{*}\right)$, a pseudo likelihood function for $\hat{\lambda}_{M}$ takes the following form

$$
L\left(\hat{\lambda}_{M} \mid \lambda_{n}\right) \propto \frac{1}{\hat{\lambda}_{M}}\left(\frac{\lambda_{n}}{\hat{\lambda}_{M}}\right)^{n^{*} / 2} \cdot \exp \left[-\frac{a \lambda_{n}}{2 \hat{\lambda}_{M}}\right], \quad \hat{\lambda}_{M}>0 .
$$

If we adopt a noninformative prior distribution of $\lambda_{n}$ (i.e., $g\left(\lambda_{n}\right) \propto 1 / \lambda_{n}$ ), then the pseudo-posterior density of $\lambda_{n}$ is

$$
f\left(\lambda_{n} \mid \hat{\lambda}_{M}\right) \propto \lambda_{n}^{n^{*} / 2-1} \cdot \exp \left[-\frac{a \lambda_{n}}{2 \hat{\lambda}_{M}}\right] .
$$

Therefore, the pseudo Bayes point estimator and the two-sided $100 \gamma \%$ Bayes CI for $\lambda_{n}$ are respectively given by

$$
\tilde{\lambda}_{n}=n^{*} \hat{\lambda}_{M} / a
$$

and

$$
\left[a^{-1} \hat{\lambda}_{M} \chi^{2}\left(n^{*} ;(1-\gamma) / 2\right), a^{-1} \hat{\lambda}_{M} \chi^{2}\left(n^{*} ;(1+\gamma) / 2\right)\right]
$$

Usually, $n^{*}$ in $(3.11)$ is not a positive integer. In this case, we can approximate $\chi^{2}\left(n^{*} ; \gamma\right)$ by

$$
\chi^{2}\left(n^{*} ; \gamma\right) \doteq n^{*}\left(1-\frac{2}{9 n^{*}}+u_{\gamma} \sqrt{\frac{2}{9 n^{*}}}\right)^{3},
$$

where $u_{\gamma}$ denotes the $\gamma$ percentage point of the standard normal distribution $\mathcal{N}(0,1)$. On the other hand, there exist algorithms to calculate $\chi^{2}\left(n^{*} ; \gamma\right)$ for fractional degrees of freedom. For example, the built-in S-plus function qchisq can handle this case.

\subsection{The Bayesian estimate of $M(t)$}

In this subsection, we consider the time-truncated case. Equivalently, we consider the posterior estimate for $\lambda_{t} \hat{=} \lambda(t)=(\beta / \alpha)(t / \alpha)^{\beta-1}$.

When $\beta$ is known, from (2.6), the posterior density of $\lambda_{t}$ is

$$
h\left(\lambda_{t} \mid Y_{\text {obs }}\right) \propto \lambda_{t}^{n-1} \exp \left(-\lambda_{t} t \beta^{-1}\right), \quad \lambda_{t}>0
$$

that is,

$$
\lambda_{t} \sim \Gamma\left(n, t \beta^{-1}\right) \quad \text { or } \quad 2 t \beta^{-1} \lambda_{t} \sim \chi^{2}(2 n) .
$$

Therefore, the Bayes point estimator and the two-sided $100 \gamma \%$ Bayes CI for $\lambda_{t}$ are respectively given by

$$
\tilde{\lambda}_{t}(\beta)=n \beta / t
$$


and

$$
\left[\frac{\beta}{2 t} \chi^{2}(2 n ;(1-\gamma) / 2), \frac{\beta}{2 t} \chi^{2}(2 n ;(1+\gamma) / 2)\right] .
$$

When $\beta$ is unknown, we consider the following transformation

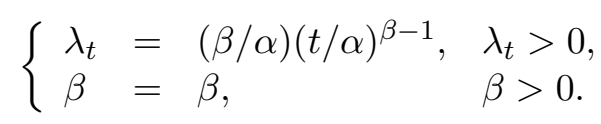

According to (2.10), the joint posterior distribution of $\left(\lambda_{t}, \beta\right)$ can be shown to be

$$
\begin{aligned}
h\left(\lambda_{t}, \beta \mid Y_{\mathrm{obs}}\right) & =h\left(\alpha, \beta \mid Y_{\mathrm{obs}}\right) \cdot J\left(\alpha, \beta \rightarrow \lambda_{t}, \beta\right) \\
& =c^{-1} \beta^{n-r}\left(u x_{r}^{r-1}\right)^{\beta} \cdot \alpha^{-n \beta-1} \exp \left[-(t / \alpha)^{\beta}\right] \cdot\left(\lambda_{t} \beta / \alpha\right)^{-1} \\
& =c^{-1} t^{n} \beta^{-r-1} \lambda_{t}^{n-1} \exp \left(-\beta z-t \lambda_{t} / \beta\right), \quad \lambda_{t}>0, \beta>0 .
\end{aligned}
$$

Integrating (3.17) with respect to $\beta$ yield the following posterior distribution of $\lambda_{t}$

$$
h\left(\lambda_{t} \mid Y_{\mathrm{obs}}\right)=c^{-1} t^{n} \lambda_{t}^{n-1} \cdot \int_{0}^{\infty} \beta^{-r-1} \exp \left(-\beta z-t \lambda_{t} / \beta\right) d \beta, \quad \lambda_{t}>0 .
$$

Therefore, the Bayes point estimator of $\lambda_{t}$ is

$$
\begin{aligned}
\tilde{\lambda}_{t} & =E\left(\lambda_{t} \mid Y_{\text {obs }}\right) \\
& =c^{-1} t^{n} \cdot \int_{0}^{\infty}\left\{\beta^{-r-1} e^{-\beta z} \int_{0}^{\infty} \lambda_{t}^{n} \exp \left(-t \lambda_{t} / \beta\right) d \lambda_{t}\right\} d \beta \\
& =n(n-r) /(t z) .
\end{aligned}
$$

From (3.17), we obtain $h\left(\lambda_{t} \mid Y_{\text {obs }}, \beta\right) \propto \lambda_{t}^{n-1} \exp \left(-t \lambda_{t} / \beta\right)$, i.e.,

$$
\lambda_{t} \mid\left(Y_{\mathrm{obs}}, \beta\right) \sim \Gamma(n, t / \beta) .
$$

To construct a Bayes CI of $\lambda_{t}$, we consider the following conditional sampling method to obtain i.i.d. posterior samples of $\lambda_{t}$.

The CONDITIONAL SAMPLING:

Step 1. Generate $m$ i.i.d. posterior samples $\left\{\beta^{(\ell)}\right\}_{\ell=1}^{m}$ of $\beta$ according to (3.6);

Step 2. For each given $\beta^{(\ell)}$, generate $\lambda_{t}^{(\ell)}$ according to $(3.20), \ell=1, \cdots, m$. $\left\{\lambda_{t}^{(\ell)}\right\}_{\ell=1}^{m}$ are then $m$ i.i.d. posterior samples of $\lambda_{t}$.

\section{Bayesian Predictions and Estimations for Single-Sample Problems}


In this section, we consider five practical single-sample issues.

\subsection{Prediction limits for $x_{n+k}$}

\subsubsection{Shape parameter $\beta$ is known}

Let $f\left(x_{n+k} \mid Y_{\text {obs }}\right)$ denote the predictive density function of the $(n+k)$-th future failure time (i.e., $x_{n+k}$ ) given the observed data $Y_{\text {obs }}$. According to (2.8), the Bayesian UPL $x_{U, B}^{(\beta)}(n, k, \gamma)$ of $x_{n+k}$ with confidence level $\gamma$ should satisfy

$$
\gamma=\operatorname{Pr}\left\{x_{n+k} \leq x_{U, B}^{(\beta)}(n, k, \gamma) \mid Y_{\mathrm{obs}}\right\}=\int_{\tau}^{x_{U, B}^{(\beta)}(n, k, \gamma)} f\left(x_{n+k} \mid Y_{\mathrm{obs}}\right) d x_{n+k},
$$

where

$$
f\left(x_{n+k} \mid Y_{\mathrm{obs}}\right)=\int_{0}^{\infty} h\left(\alpha \mid Y_{\mathrm{obs}}\right) \cdot f\left(x_{n+k} \mid Y_{\mathrm{obs}}, \alpha\right) d \alpha .
$$

It should be noted that $h\left(\alpha \mid Y_{\text {obs }}\right)$ is given by (2.6) and

$$
\begin{aligned}
f\left(x_{n+k} \mid Y_{\mathrm{obs}}, \alpha\right) & =\frac{f\left(Y_{\mathrm{obs}}, x_{n+k} \mid \alpha\right)}{f\left(Y_{\mathrm{obs}} \mid \alpha\right)} \\
& =\frac{\int_{\tau<x_{n+1}<\cdots<x_{n+k-1}<x_{n+k}} f\left(x_{r}, x_{r+1}, \cdots, x_{n+k} \mid \alpha\right) \prod_{i=n+1}^{n+k-1} d x_{i}}{f\left(Y_{\mathrm{obs}} \mid \alpha\right)},
\end{aligned}
$$

where $f\left(Y_{\text {obs }} \mid \alpha\right)$ is given by $(2.3)$ and $f\left(x_{r}, x_{r+1}, \cdots, x_{n+k} \mid \alpha\right)$ is also given by (2.3) with $(n, \tau)$ being replaced by $\left(n+k, x_{n+k}\right)$, that is,

$$
f\left(x_{r}, x_{r+1}, \cdots, x_{n+k} \mid \alpha\right)=\frac{\beta^{n+k-r+1} \exp \left[-\left(x_{n+k} / \alpha\right)^{\beta}\right]}{(r-1) ! \alpha^{(n+k) \beta}} \cdot x_{r}^{(r-1) \beta} \prod_{i=r}^{n+k} x_{i}^{\beta-1} .
$$

By using the identity (2.2) in Yu et al. (2008), we immediately have the numerator in the right-hand side of (4.3) being equal to

$$
\frac{\beta^{n-r+2} \exp \left[-\left(x_{n+k} / \alpha\right)^{\beta}\right]}{(r-1) !(k-1) ! \alpha^{(n+k) \beta}} \cdot x_{r}^{(r-1) \beta} u^{\beta-1} \cdot x_{n+k}^{\beta-1}\left[x_{n+k}^{\beta}-\tau^{\beta}\right]^{k-1} \text {. }
$$

Hence, (4.3) (cf. Calabria, Guida and Pulcini, 1990) and (4.2) become

$$
f\left(x_{n+k} \mid Y_{\text {obs }}, \alpha\right)=\frac{\beta \exp \left[-\left(x_{n+k}^{\beta}-\tau^{\beta}\right) / \alpha^{\beta}\right]}{(k-1) ! \alpha^{k \beta}} \cdot x_{n+k}^{\beta-1}\left[x_{n+k}^{\beta}-\tau^{\beta}\right]^{k-1},
$$

and

$$
f\left(x_{n+k} \mid Y_{\mathrm{obs}}\right)=\frac{1}{B(n, k)} \cdot \frac{\beta}{x_{n+k}}\left(\frac{\tau}{x_{n+k}}\right)^{n \beta}\left[1-\left(\frac{\tau}{x_{n+k}}\right)^{\beta}\right]^{k-1},
$$


respectively. Substituting (4.5) into (4.1) yields

$$
\gamma=\frac{1}{B(n, k)} \int_{\left[\tau / x_{U, B}^{(\beta)}(n, k, \gamma)\right]^{\beta}}^{1} y^{n-1}(1-y)^{k-1} d y .
$$

Thus, $\left[\tau / x_{U, B}^{(\beta)}(n, k, \gamma)\right]^{\beta}$ equals to the $1-\gamma$ percentage point of the $\operatorname{Beta}(n, k)$ distribution. From the relationship between the quantiles of beta distribution and $F$-distribution, we have

$$
x_{U, B}^{(\beta)}(n, k, \gamma)=\tau\left[\frac{k}{n} F(2 k, 2 n ; \gamma)+1\right]^{1 / \beta},
$$

where $F(m, n ; \gamma)$ represents the $\gamma$ percentage point of the $F$-distribution with $m$ and $n$ degrees of freedom.

\subsubsection{Shape parameter $\beta$ is unknown}

The Bayes UPL for $x_{n+k}$ with confidence level $\gamma$ satisfies

$$
\begin{aligned}
\gamma & =\int_{\tau}^{x_{U, B}(n, k, r, \gamma)} f\left(x_{n+k} \mid Y_{\mathrm{obs}}\right) d x_{n+k} \\
& =\int_{\tau}^{x_{U, B}(n, k, r, \gamma)}\left\{\int_{0}^{\infty} \int_{0}^{\infty} h\left(\alpha, \beta \mid Y_{\mathrm{obs}}\right) \cdot f\left(x_{n+k} \mid Y_{\mathrm{obs}}, \alpha, \beta\right) d \alpha d \beta\right\} d x_{n+k}
\end{aligned}
$$

where $h\left(\alpha, \beta \mid Y_{\text {obs }}\right)$ is given in (2.10) while $f\left(x_{n+k} \mid Y_{\text {obs }}, \alpha, \beta\right)$ is given in (4.4). Thus,

$$
\begin{aligned}
& \int_{0}^{\infty} \int_{0}^{\infty} h\left(\alpha, \beta \mid Y_{\mathrm{obs}}\right) \cdot f\left(x_{n+k} \mid Y_{\mathrm{obs}}, \alpha, \beta\right) d \alpha d \beta \\
= & \frac{\Gamma(n+k)}{c(k-1) !} \cdot \int_{0}^{\infty} \beta^{n-r} e^{-\beta z} \frac{1}{x_{n+k}}\left(\frac{\tau}{x_{n+k}}\right)^{n \beta}\left[1-\left(\frac{\tau}{x_{n+k}}\right)^{\beta}\right]^{k-1} d \beta .
\end{aligned}
$$

It can be shown that

$$
x_{U, B}(n, k, r, \gamma)=\tau \exp \left\{y_{\gamma} \cdot z /[(n-r)(n-r+1)]\right\},
$$

where $y_{\gamma}$ is the solution to the following equation

$$
\gamma=\sum_{j=1}^{k}\left(\prod_{i=1, i \neq j}^{k} \frac{n+k-i}{j-i}\right) \cdot\left[1-\left(1+\frac{(n+k-j) y_{\gamma}}{(n-r)(n-r+1)}\right)^{-n+r}\right] .
$$


To prove (4.9) and (4.10), we substitute both (4.9) and (4.8) into (4.7) and then make the transformation $x=\left(\tau / x_{n+k}\right)^{\beta}$, which yields

$$
\gamma=\frac{\Gamma(n+k)}{c(k-1) !} \cdot \int_{0}^{\infty} \beta^{n-r-1} e^{-\beta z}\left\{\int_{R}^{1} x^{n-1}(1-x)^{k-1} d x\right\} d \beta
$$

where $R=e^{-\beta y_{\gamma} z /[(n-r)(n-r+1)]}$. Using the following identity

$$
\frac{1}{B(n, k)} \int_{R}^{1} x^{n-1}(1-x)^{k-1} d x=1-\sum_{j=1}^{k}\left(\begin{array}{c}
n+k-1 \\
j-1
\end{array}\right) R^{n+k-j}(1-R)^{j-1}
$$

and expanding the term $(1-R)^{j-1}$, we can readily obtain (4.10).

\subsection{Estimating the probability of $N(\tau, T) \leq k$}

Based on $Y_{\mathrm{obs}}^{\mathrm{ft}}$ or $Y_{\mathrm{obs}}^{\mathrm{tt}}$, we are interested in the following question:

IssuE A1: What is the probability that at most $k$ failures will occur in the future time period $(\tau, T]$ with $T>\tau$ ?

Equivalently, we wish to estimate the following probability:

$$
\gamma_{k}=\operatorname{Pr}\left\{N(\tau, T) \leq k \mid Y_{\text {obs }}\right\}=\operatorname{Pr}\left\{N(T) \leq n+k \mid Y_{\text {obs }}\right\},
$$

where $N(\tau, T) \hat{=} N(T)-N(\tau)=N(T)-n$.

\subsubsection{Shape parameter $\beta$ is known}

In this case, we denote $\gamma_{k}$ in (4.11) by $\gamma_{k}^{(\beta)}$ and we have

$$
\begin{aligned}
\gamma_{k}^{(\beta)} & =\int_{0}^{\infty} h\left(\alpha \mid Y_{\text {obs }}\right) \cdot \operatorname{Pr}\left\{N(T) \leq n+k \mid Y_{\text {obs }}, \alpha\right\} d \alpha \\
& =\int_{0}^{\infty} h\left(\alpha \mid Y_{\text {obs }}\right) \cdot \sum_{j=n}^{n+k} \operatorname{Pr}\left\{N(T)=j \mid Y_{\text {obs }}, \alpha\right\} d \alpha \\
& =\int_{0}^{\infty} h\left(\alpha \mid Y_{\text {obs }}\right) \cdot \sum_{j=n}^{n+k} \frac{f\left(Y_{\text {obs }}, N(T)=j \mid \alpha\right)}{f\left(Y_{\text {obs }} \mid \alpha\right)} d \alpha
\end{aligned}
$$


where $h\left(\alpha \mid Y_{\text {obs }}\right)$ and $f\left(Y_{\text {obs }} \mid \alpha\right)$ are respectively given by (2.6) and (2.3), and

$$
\begin{aligned}
& f\left(Y_{\mathrm{obs}}, N(T)=j \mid \alpha\right) \\
= & \int_{\tau<x_{n+1}<\cdots<x_{j}<T} f\left(Y_{\mathrm{obs}}, x_{n+1}, \cdots, x_{j}, N(T)=j \mid \alpha\right) \prod_{\ell=n+1}^{j} d x_{\ell} \\
\stackrel{(2.3)}{=} & \int_{\tau<x_{n+1}<\cdots<x_{j}<T} \frac{\beta^{j-r+1} e^{-(T / \alpha)^{\beta}} \cdot x_{r}^{(r-1) \beta} \prod_{i=r}^{j} x_{i}^{\beta-1}}{(r-1) ! \alpha^{j \beta}} \prod_{\ell=n+1}^{j} d x_{\ell} \\
= & \frac{\beta^{n-r+1} e^{-(T / \alpha)^{\beta}} \cdot x_{r}^{(r-1) \beta} u^{\beta-1}\left[T^{\beta}-\tau^{\beta}\right]^{j-n}}{(r-1) !(j-n) ! \alpha^{j \beta}} .
\end{aligned}
$$

Substituting (4.13) into (4.12) yields ${ }^{2}$

$$
\begin{aligned}
\gamma_{k}^{(\beta)} & =\sum_{j=n}^{n+k} \int_{0}^{\infty} \frac{\beta \tau^{n \beta}\left[T^{\beta}-\tau^{\beta}\right]^{j-n}}{\Gamma(n)(j-n) !} \cdot \alpha^{-j \beta-1} e^{-(T / \alpha)^{\beta}} d \alpha \\
& =\sum_{j=n}^{n+k}\left(\begin{array}{l}
j-1 \\
n-1
\end{array}\right) \delta^{n}(1-\delta)^{j-n} \\
& =\left(\frac{\delta}{1-\delta}\right)^{n} \sum_{j=n}^{n+k}\left(\begin{array}{l}
j-1 \\
n-1
\end{array}\right)(1-\delta)^{j},
\end{aligned}
$$

where

$$
\delta \hat{=}(\tau / T)^{\beta}
$$

In particular, from (4.14), we have the recursive formula

$$
\gamma_{0}^{(\beta)}=\delta^{n}, \quad \gamma_{1}^{(\beta)}=\delta^{n}(n+1-n \delta),
$$

and

$$
\gamma_{k}^{(\beta)}=\gamma_{k-1}^{(\beta)}+\left(\begin{array}{c}
n+k-1 \\
n-1
\end{array}\right)(1-\delta)^{k} \delta^{n}, \quad k \geq 2
$$

\subsubsection{Shape parameter $\beta$ is unknown}

${ }^{2}$ Alternatively, formula (4.14) can also be obtained by exploiting the following result:

$$
\operatorname{Pr}\left\{N(\tau, T) \leq k \mid Y_{\text {obs }}, \alpha, \beta\right\}=\sum_{i=0}^{k} \frac{\left[(T / \alpha)^{\beta}-(\tau / \alpha)^{\beta}\right]^{i}}{i !} \cdot \exp \left[-(T / \alpha)^{\beta}+(\tau / \alpha)^{\beta}\right]
$$


In this case, (4.11) becomes

$$
\begin{aligned}
\gamma_{k} & =\int_{0}^{\infty} \int_{0}^{\infty} h\left(\alpha, \beta \mid Y_{\mathrm{obs}}\right) \cdot \operatorname{Pr}\left\{N(T) \leq n+k \mid Y_{\mathrm{obs}}, \alpha, \beta\right\} d \alpha d \beta \\
& =\int_{0}^{\infty} \int_{0}^{\infty} h\left(\alpha, \beta \mid Y_{\mathrm{obs}}\right) \cdot \sum_{j=n}^{n+k} \frac{f\left(Y_{\mathrm{obs}}, N(T)=j \mid \alpha, \beta\right)}{f\left(Y_{\mathrm{obs}} \mid \alpha, \beta\right)} d \alpha d \beta
\end{aligned}
$$

where $h\left(\alpha, \beta \mid Y_{\text {obs }}\right), f\left(Y_{\text {obs }} \mid \alpha, \beta\right)$ and $f\left(Y_{\text {obs }}, N(T)=j \mid \alpha, \beta\right)$ are respectively given in (2.10), (2.3) and (4.13). Thus, we have

$$
\begin{aligned}
\gamma_{k} & =\sum_{j=n}^{n+k} \frac{z^{n-r} \Gamma(j)}{\Gamma(n) \Gamma(n-r)(j-n) !} \cdot \int_{0}^{\infty} \beta^{n-r-1}\left(u x_{r}^{r-1} / T^{n}\right)^{\beta}\left[1-(\tau / T)^{\beta}\right]^{j-n} d \beta \\
& =\sum_{j=n}^{n+k} \sum_{i=0}^{j-n} \frac{z^{n-r} \Gamma(j)(-1)^{i}}{(n-1) !(j-n-i) ! i !}[z+(n+i) \ln (T / \tau)]^{-n+r}
\end{aligned}
$$

In particular,

$$
\gamma_{0}=\left[\frac{z}{z+n \ln (T / \tau)}\right]^{n-r}
$$

and

$$
\gamma_{1}=(n+1) \gamma_{0}-n\left[\frac{z}{z+(n+1) \ln (T / \tau)}\right]^{n-r} \text {. }
$$

\subsection{Estimating the probability of $\lambda(T) \leq \lambda_{0}$}

Based on $Y_{\mathrm{obs}}^{\mathrm{ft}}$ or $Y_{\mathrm{obs}}^{\mathrm{tt}}$, we are interested in the following question

Issue B1: Given that a pre-determined target value $\lambda_{0}$ for the failure rate of the system undergoing development testing is not achieved at time $\tau$, what is the probability that the target $\lambda_{0}$ will be achieved at time $T$ with $T>\tau$ ?

Equivalently, we wish to estimate the following probability:

$$
\gamma=\operatorname{Pr}\left\{\lambda(T) \leq \lambda_{0} \mid Y_{\text {obs }}\right\}
$$

where $\lambda(T)=(\beta / \alpha)(T / \alpha)^{\beta-1} \hat{=} \lambda_{T}$.

\subsubsection{Shape parameter $\beta$ is known}

Similar to (3.14), the posterior density of $\lambda_{T}$ can be easily shown to be

$$
h\left(\lambda_{T} \mid Y_{\mathrm{obs}}\right) \propto \lambda_{T}^{n-1} \exp \left[-\lambda_{T} \cdot \tau^{\beta}\left(\beta T^{\beta-1}\right)^{-1}\right], \quad \lambda_{T}>0 .
$$


Substituting (4.22) into (4.21) and using the following relationship between the gamma and Poisson distribution functions

$$
\frac{b^{a}}{\Gamma(a)} \int_{0}^{\lambda} x^{a-1} e^{-x b} d x=1-\sum_{h=0}^{a-1} \frac{(b \lambda)^{h}}{h !} e^{-b \lambda},
$$

we have

$$
\begin{aligned}
\gamma^{(\beta)} & =\int_{0}^{\lambda_{0}} h\left(\lambda_{T} \mid Y_{\mathrm{obs}}\right) d \lambda_{T} \\
& =1-\sum_{h=0}^{n-1} \frac{\left[\tau^{\beta}\left(\beta T^{\beta-1}\right)^{-1} \lambda_{0}\right]^{h}}{h !} \exp \left[-\tau^{\beta}\left(\beta T^{\beta-1}\right)^{-1} \lambda_{0}\right] \\
& =1-\sum_{h=0}^{n-1} \operatorname{Poisson}(h \mid \theta(\beta)),
\end{aligned}
$$

where $\theta(\beta) \hat{=} \tau^{\beta}\left(\beta T^{\beta-1}\right)^{-1} \lambda_{0}$ and $\operatorname{Poisson}(h \mid \theta) \hat{=} \theta^{h} e^{-\theta} / h$ !

\subsubsection{Shape parameter $\beta$ is unknown}

Consider the following transformation

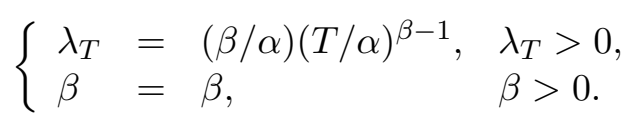

Similar to (3.17) and (3.18), the posterior distribution of $\lambda_{T}$ is

$$
h\left(\lambda_{T} \mid Y_{\mathrm{obs}}\right)=\frac{z^{n-r}}{\Gamma(n) \Gamma(n-r)} \int_{0}^{\infty} \frac{\beta^{n-r-1}\left(u x_{r}^{r-1}\right)^{\beta}}{\left(\beta T^{\beta-1}\right)^{n}} \cdot \lambda_{T}^{n-1} \exp \left[-\tau^{\beta}\left(\beta T^{\beta-1}\right)^{-1} \lambda_{T}\right] d \beta .
$$

Substituting this expression into (4.21), we have

$$
\begin{aligned}
\gamma & =\int_{0}^{\lambda_{0}} h\left(\lambda_{T} \mid Y_{\mathrm{obs}}\right) d \lambda_{T} \\
& =1-\sum_{h=0}^{n-1} \int_{0}^{\infty} \frac{[\theta(\beta)]^{h}}{h !} e^{-\theta(\beta)} \cdot \frac{z^{n-r}}{\Gamma(n-r)} \beta^{n-r-1} e^{-\beta z} d \beta \\
& =1-\sum_{h=0}^{n-1} \int_{0}^{\infty} \operatorname{Poisson}(h \mid \theta(\beta)) \cdot \frac{z^{n-r}}{\Gamma(n-r)} \beta^{n-r-1} e^{-\beta z} d \beta
\end{aligned}
$$

\subsection{Predicting the time $T$ required to achieve the target failure-rate}


In this subsection, we are interested in the following issue:

Issue C1: Given that the target value $\lambda_{0}$ for the system failure-rate is not achieved at $\tau$, how long will it take in order that the system failure rate will be attained at $\lambda_{0}$ ?

Statistically, for a given confidence level $\gamma$ and the target failure-rate $\lambda_{0}$, we wish to estimate the time $T(T>\tau)$ that satisfies (4.21). When $\beta$ is known, it is easy to see from (4.22) that

$$
2 \tau^{\beta}\left(\beta T^{\beta-1}\right)^{-1} \lambda_{T} \mid Y_{\text {obs }} \sim \chi^{2}(2 n) .
$$

From (4.21), we have

$$
\gamma=\operatorname{Pr}\left\{2 \tau^{\beta}\left(\beta T^{\beta-1}\right)^{-1} \lambda_{T} \leq 2 \tau^{\beta}\left(\beta T^{\beta-1}\right)^{-1} \lambda_{0} \mid Y_{\text {obs }}\right\},
$$

which implies $2 \tau^{\beta}\left(\beta T^{\beta-1}\right)^{-1} \lambda_{0}=\chi^{2}(2 n ; \gamma)$ or

$$
T=\left[\frac{2 \lambda_{0} \tau^{\beta}}{\beta \chi^{2}(2 n ; \gamma)}\right]^{1 /(\beta-1)} .
$$

When $\beta$ is unknown, the desired $T$ that satisfies (4.25) can be solved by using an iterative algorithm.

Equivalently, we notice that Issue C1 can be re-formulated as follows:

Issue D1: Based on $Y_{\text {obs }}^{f t}$ or $Y_{\text {obs }}^{t t}$, what is the Bayes UPL of $\lambda(T)$ with $T$ being a pre-specified value larger than $\tau$ ?

Statistically, it is desired to find $\lambda_{U, B}(T)$ satisfying $\gamma=\operatorname{Pr}\left\{\lambda(T) \leq \lambda_{U, B}(T) \mid Y_{\text {obs }}\right\}$ for a given level $\gamma$. When $\beta$ is known, solving $\lambda_{0}$ from (4.26), we obtain

$$
\lambda_{U, B}(T)=\frac{\beta T^{\beta-1} \chi^{2}(2 n ; \gamma)}{2 \tau^{\beta}} .
$$

When $\beta$ is unknown, the corresponding $\lambda_{U, B}(T)$ equals to the $\lambda_{0}$ satisfying (4.25). Thus, an iterative algorithm can be applied.

\section{Bayesian Predictions and Estimations for Two-Sample Problems}

Suppose that the successive failure times of two repairable systems follow the same PLP with intensity function (1.1). Furthermore, for the first system, we assume that the first $(r-1)$ failure times were missing and the remainder failure times (i.e., $Y_{\mathrm{obs}}^{\mathrm{ft}}$ or $Y_{\mathrm{obs}}^{\mathrm{tt}}$ ) have been recorded exactly. We are interested in the following two-sample problems. 
Issue A2: How to predict the $k$-th $(k \geq 1)$ failure time $y_{k}$ of the second system?

Issue B2: Assume that the number of failures in the time interval $\left(0, t_{2}\right]$ for the second system is $m$ but that the exact failure times are unknown, how to predict the $k$-th $(1 \leq k \leq m)$ failure time $y_{k}$ of the second system?

Issue C2: What is the probability that at most $m$ failures will occur in $\left(0, t_{2}\right]$ for the second system?

In this section, we utilize a Bayesian approach to address the above three issues.

\subsection{Prediction limits for the $k$-th failure time of the 2-nd system}

This subsection considers Issue A2. We first note that $2\left(x_{n} / \alpha\right)^{\beta} \sim \chi^{2}(2 n)$ (Bain, 1978; Theorem 1 of Yu et al., 2008). By replacing $x_{n}$ by $y_{k}$, for the second system, we have $2\left(y_{k} / \alpha\right)^{\beta} \sim \chi^{2}(2 k)$ or $\left(y_{k} / \alpha\right)^{\beta} \sim \Gamma(k, 1)$. Thus, the sampling distribution of $y_{k}$ from a Bayesian viewpoint is given by

$$
f\left(y_{k}\right)=f\left(y_{k} \mid \alpha\right)=f\left(y_{k} \mid \alpha, \beta\right)=\frac{1}{\Gamma(k)} \beta \alpha^{-k \beta} y_{k}^{k \beta-1} \exp \left[-\left(y_{k} / \alpha\right)^{\beta}\right] .
$$

\subsubsection{Shape parameter $\beta$ is known}

The predictive density of the $k$-th failure time $y_{k}$ of the second system is

$$
\begin{aligned}
f\left(y_{k} \mid Y_{\mathrm{obs}}\right) & =\int_{0}^{\infty} h\left(\alpha \mid Y_{\mathrm{obs}}\right) f\left(y_{k} \mid Y_{\mathrm{obs}}, \alpha\right) d \alpha \\
& =\int_{0}^{\infty} h\left(\alpha \mid Y_{\mathrm{obs}}\right) f\left(y_{k} \mid \alpha\right) d \alpha \quad \text { by the independence of } y_{k} \text { and } Y_{\mathrm{obs}} \\
& =\frac{\beta \tau^{n \beta}}{B(n, k)} \cdot \frac{y_{k}^{k \beta-1}}{\left(\tau^{\beta}+y_{k}^{\beta}\right)^{n+k}} . \quad \text { by (2.6) and (5.1) }
\end{aligned}
$$

Hence, the Bayes UPL $y_{U, B}^{(\beta)}(k, n, \gamma)$ for $y_{k}$ with confidence level $\gamma$ satisfies

$$
\begin{array}{rlr}
\gamma & =\int_{0}^{y_{U, B}^{(\beta)}(k, n, \gamma)} f\left(y_{k} \mid Y_{\mathrm{obs}}\right) d y_{k} & \text { by }(5.2) \\
& =\int_{0}^{y_{U, B}^{(\beta)}(k, n, \gamma)} \frac{\beta \tau^{n \beta}}{B(n, k)} \cdot \frac{y_{k}^{k \beta-1}}{\left(\tau^{\beta}+y_{k}^{\beta}\right)^{n+k}} d y_{k} & \text { let } y=\frac{n y_{k}^{\beta}}{k \tau^{\beta}} \\
& =\int_{0}^{n\left[y_{U, B}^{(\beta)}(k, n, \gamma)\right]^{\beta} /\left(k \tau^{\beta}\right)} \frac{1}{B(n, k)}\left(\frac{k}{n}\right)^{k} y^{k-1}\left(1+\frac{k}{n} y\right)^{-n-k} d y .
\end{array}
$$


Note that the integrand in the above integration is exactly the pdf of the $F(2 k, 2 n)$ distribution; thus $n\left[y_{U, B}^{(\beta)}(k, n, \gamma)\right]^{\beta} /\left(k \tau^{\beta}\right)=F(2 k, 2 n ; \gamma)$ and

$$
y_{U, B}^{(\beta)}(k, n, \gamma)=\tau\left[\frac{k}{n} F(2 k, 2 n ; \gamma)\right]^{1 / \beta} .
$$

\subsubsection{Shape parameter $\beta$ is unknown}

Note that the independency of $y_{k}$ and $Y_{\text {obs }}$, then, the predictive density of $y_{k}$ is

$$
\begin{aligned}
f\left(y_{k} \mid Y_{\mathrm{obs}}\right) & =\int_{0}^{\infty} \int_{0}^{\infty} h\left(\alpha, \beta \mid Y_{\mathrm{obs}}\right) f\left(y_{k} \mid Y_{\mathrm{obs}}, \alpha, \beta\right) d \alpha d \beta \\
& =\int_{0}^{\infty} \int_{0}^{\infty} h\left(\alpha, \beta \mid Y_{\mathrm{obs}}\right) f\left(y_{k} \mid \alpha, \beta\right) d \alpha d \beta \\
& =\frac{\Gamma(n+k)}{c \Gamma(k)} \cdot \int_{0}^{\infty} \beta^{n-r}\left(u x_{r}^{r-1}\right)^{\beta} \frac{y_{k}^{k \beta-1}}{\left(\tau^{\beta}+y_{k}^{\beta}\right)^{n+k}} d \beta .
\end{aligned}
$$

Thus, the Bayes UPL $y_{U, B}(k, n, r, \gamma)$ for $y_{k}$ satisfies

$$
\begin{aligned}
\gamma & =\int_{0}^{y_{U, B}(k, n, r, \gamma)} f\left(y_{k} \mid Y_{\text {obs }}\right) d y_{k} \\
& =\frac{z^{n-r}}{B(n, k) \Gamma(n-r)} \int_{0}^{\infty} \beta^{n-r}\left(u x_{r}^{r-1}\right)^{\beta} \int_{0}^{y_{U, B}(k, n, \gamma)} \frac{y_{k}^{k \beta-1}}{\left(\tau^{\beta}+y_{k}^{\beta}\right)^{n+k}} d y_{k} d \beta .
\end{aligned}
$$

It is easy to show that

$$
y_{U, B}(k, n, r, \gamma)=\tau V^{z /(n-r+1)},
$$

where $V$ is the solution to the following equation

$$
\begin{aligned}
\gamma & =\int_{0}^{\infty} F\left(n k^{-1} V^{\beta z /(n-r+1)} \mid 2 k, 2 n\right) \cdot \frac{z^{n-r}}{\Gamma(n-r)} \beta^{n-r-1} e^{-\beta z} d \beta \\
& =\int_{0}^{\infty} F\left(n k^{-1} V^{x /(n-r+1)} \mid 2 k, 2 n\right) \cdot \chi^{2}(x \mid 2 n-2 r) d x
\end{aligned}
$$

with $F(\cdot \mid m, n)$ and $\chi^{2}(\cdot \mid n)$ being the cdf of $F(m, n)$ and the density of $\chi^{2}(n)$, respectively.

\subsection{Prediction limits for $y_{k}$ given $N\left(t_{2}\right)=m$}


For Issue B2, we first need to find the conditional density $f\left(y_{k} \mid N\left(t_{2}\right)=m\right)$. Setting $r=1$ and replacing $n, t$, and $x_{i}$ by $m, t_{2}$ and $y_{i}$ respectively in (2.2), we obtain

$$
f\left(y_{1}, \cdots, y_{m} ; N\left(t_{2}\right)=m\right)=\beta^{m} \alpha^{-m \beta}\left(\prod_{i=1}^{m} y_{i}^{\beta-1}\right) \exp \left[-\left(t_{2} / \alpha\right)^{\beta}\right] .
$$

By integrating out the variables $y_{1}, \cdots, y_{k-1}, y_{k+1}, \cdots, y_{m}$ from the above joint density, we have

$$
f\left(y_{k} ; N\left(t_{2}\right)=m\right)=\frac{\beta y_{k}^{k \beta-1}\left[t_{2}^{\beta}-y_{k}^{\beta}\right]^{m-k}}{(k-1) !(m-k) ! \alpha^{m \beta}} \cdot \exp \left[-\left(t_{2} / \alpha\right)^{\beta}\right]
$$

and

$$
\begin{aligned}
f\left(y_{k} \mid N\left(t_{2}\right)=m\right) & =\frac{f\left(y_{k} ; N\left(t_{2}\right)=m\right)}{\operatorname{Pr}\left\{N\left(t_{2}\right)=m\right\}} \\
& =\frac{1}{B(k, m-k+1)} \beta y_{k}^{k \beta-1} t_{2}^{-m \beta}\left[t_{2}^{\beta}-y_{k}^{\beta}\right]^{m-k}, \quad y_{k}<t_{2} .
\end{aligned}
$$

It is noteworthy that (5.7) does not depend on $\alpha$.

\subsubsection{Shape parameter $\beta$ is known} have

Since $y_{k}$ is independent of the observed data $Y_{\text {obs }}$ for the first system, we also

$$
f\left(y_{k} \mid N\left(t_{2}\right)=m, Y_{\text {obs }}\right)=f\left(y_{k} \mid N\left(t_{2}\right)=m\right) .
$$

Given $N\left(t_{2}\right)=m$, the Bayes UPL $y_{U, B}^{(\beta)}(k, m, \gamma)$ for $y_{k}$ with level $\gamma$ satisfies

$$
\begin{aligned}
\gamma & =\int_{0}^{y_{U, B}^{(\beta)}(k, m, \gamma)} f\left(y_{k} \mid N\left(t_{2}\right)=m, Y_{\mathrm{obs}}\right) d y_{k} \\
& =\frac{1}{B(k, m-k+1)} \int_{0}^{\left[y_{U, B}^{(\beta)}(k, m, \gamma) / t_{2}\right]^{\beta}} x^{k-1}(1-x)^{m-k} d x .
\end{aligned}
$$

Hence, $\left[y_{U, B}^{(\beta)}(k, m, \gamma) / t_{2}\right]^{\beta}$ is equal to the $\gamma$ percentage point of the $\operatorname{Beta}(k, m-$ $k+1)$ distribution. Similar to (4.6), we obtain

$$
y_{U, B}^{(\beta)}(k, m, \gamma)=t_{2}\left[1+\frac{m-k+1}{k} F^{-1}(2 k, 2 m-2 k+2 ; \gamma)\right]^{-1 / \beta} \text {. }
$$

\subsubsection{Shape parameter $\beta$ is unknown}


From (2.10) and (5.7), we have

$$
\begin{aligned}
& f\left(y_{k} \mid N\left(t_{2}\right)=m, Y_{\mathrm{obs}}\right) \\
= & \int_{0}^{\infty} \int_{0}^{\infty} f\left(y_{k} \mid N\left(t_{2}\right)=m\right) \cdot h\left(\alpha, \beta \mid Y_{\mathrm{obs}}\right) d \alpha a \beta \\
= & \frac{z^{n-r}}{B(k, m-k+1) \Gamma(n-r)} \int_{0}^{\infty} \beta^{n-r} e^{-\beta z} t_{2}^{-k \beta} y_{k}^{k \beta-1}\left[1-\left(y_{k} / t_{2}\right)^{\beta}\right]^{m-k} d \beta \\
= & \frac{\Gamma(n-r+1) z^{n-r}}{B(k, m-k+1) \Gamma(n-r)} \sum_{i=0}^{m-k}(-1)^{i}\left(\begin{array}{c}
m-k \\
i
\end{array}\right) y_{k}^{-1}\left[z+(k+i) \ln \left(t_{2} / y_{k}\right)\right]^{-(n-r+1)} .
\end{aligned}
$$

The Bayes UPL $y_{U, B}(k, m, n, r, \gamma)$ for $y_{k}$ with level $\gamma$ satisfies

$$
\begin{aligned}
\gamma & =\int_{0}^{y_{U, B}(k, m, n, r, \gamma)} f\left(y_{k} \mid N\left(t_{2}\right)=m, Y_{\mathrm{obs}}\right) d y_{k} \\
& =\frac{1}{B(k, m-k+1)} \sum_{i=0}^{m-k} \frac{(-1)^{i}}{k+i}\left(\begin{array}{c}
m-k \\
i
\end{array}\right)\left[1+\frac{k+i}{z} \ln \frac{t_{2}}{y_{U, B}(k, m, n, r, \gamma)}\right]^{-(n-r)} .
\end{aligned}
$$

Therefore,

$$
y_{U, B}(k, m, n, r, \gamma)=t_{2} e^{-z W},
$$

where $W$ can be determined by

$$
\gamma=\frac{1}{B(k, m-k+1)} \sum_{i=0}^{m-k} \frac{(-1)^{i}}{k+i}\left(\begin{array}{c}
m-k \\
i
\end{array}\right)[1+(k+i) W]^{-(n-r)} .
$$

In particular, when $k=m$ we have $W=\left(\gamma^{-\frac{1}{n-r}}-1\right) / m$ and

$$
y_{U, B}(m, m, n, r, \gamma)=t_{2} \exp \left[-\frac{z}{m}\left(\gamma^{-\frac{1}{n-r}}-1\right)\right] .
$$

\subsection{Estimating the probability of $N\left(t_{2}\right) \leq m$}

For Issue $\mathrm{C} 2$, the probability that at most $m$ (a pre-specified value) failures will occur in $\left(0, t_{2}\right]$ for the second system is given by

$$
\gamma_{m}=\operatorname{Pr}\left\{N\left(t_{2}\right) \leq m \mid Y_{\text {obs }}\right\}=\sum_{k=0}^{m} \operatorname{Pr}\left\{N\left(t_{2}\right)=k \mid Y_{\text {obs }}\right\} .
$$

Note that

$$
\operatorname{Pr}\left\{N\left(t_{2}\right)=k\right\}=\frac{\left(t_{2} / \alpha\right)^{k \beta}}{k !} \exp \left[-\left(t_{2} / \alpha\right)^{\beta}\right]
$$


When $\beta$ is known, we have

$$
\begin{aligned}
\operatorname{Pr}\left\{N\left(t_{2}\right)=k \mid Y_{\text {obs }}\right\} & =\int_{0}^{\infty} \operatorname{Pr}\left\{N\left(t_{2}\right)=k \mid \alpha\right\} \cdot h\left(\alpha \mid Y_{\text {obs }}\right) d \alpha \\
& =\int_{0}^{\infty} \operatorname{Pr}\left\{N\left(t_{2}\right)=k\right\} \cdot h\left(\alpha \mid Y_{\text {obs }}\right) d \alpha \\
& =\left(\begin{array}{c}
n+k-1 \\
k
\end{array}\right) q^{k}(1-q)^{n} .
\end{aligned}
$$

Substituting this into (5.12), we obtain

$$
\gamma_{m}^{(\beta)}=(1-q)^{n} \sum_{k=0}^{m}\left(\begin{array}{c}
n+k-1 \\
k
\end{array}\right) q^{k},
$$

where

$$
q \hat{=} t_{2}^{\beta} /\left[\tau^{\beta}+t_{2}^{\beta}\right]
$$

When $\beta$ is unknown, we have

$$
\begin{aligned}
\gamma_{m} & =\sum_{k=0}^{m} \operatorname{Pr}\left\{N\left(t_{2}\right)=k \mid Y_{\mathrm{obs}}\right\} \\
& =\sum_{k=0}^{m} \int_{0}^{\infty} \int_{0}^{\infty} \operatorname{Pr}\left\{N\left(t_{2}\right)=k\right\} \cdot h\left(\alpha, \beta \mid Y_{\mathrm{obs}}\right) d \alpha d \beta \\
& =\frac{z^{n-r}}{\Gamma(n-r)} \int_{0}^{\infty} \beta^{n-r-1} e^{-\beta z} \gamma_{m}^{(\beta)} d \beta,
\end{aligned}
$$

where $\gamma_{m}^{(\beta)}$ is given in (5.13).

\section{Numerical Examples}

In this section, two real examples from an engine development program and a repairable system are used to illustrate the proposed methodologies.

\subsection{Engine failure data}

Zhou \& Weng (1992, p.51-52) reported a total of 40 failures for an engine undergoing development testing in the time interval $(0,8063]$. The data are given by $*, *, *, 171,234,274,377,530,533,941,1074,1188,1248,2298,2347$, 2347, 2381, 2456, 2456, 2500, 2913, 3022, 3038, 3728, 3873, 4724, 5147, 5179, 5587, 5626, 6824, 6983, 7106, 7106, 7568, 7568, 7593, 7642, 7928, 8063 hours. Here, the exact failure times for the first three failures are unknown and are 
denoted by $*$. Since these data are failure-truncated, we have $n=40, \tau=x_{40}$ $=8063$ and $r=4$. Yu, Tian \& Tang (2008) performed a goodness-of-fit test which fails to reject the hypothesis that the data come from a PLP the the 0.05 significant level with the MLE of $\beta$ being $\hat{\beta}=0.6761$.

We first consider the case that $\beta$ is known by letting $\beta=\hat{\beta}=0.6761$. From (3.3) and (3.4), the Bayes point estimator of $\alpha$ is $\tilde{\alpha}(\beta)=36.0680$ and the twosided $95 \%$ Bayes CI for $\alpha$ is $[22.5067,56.6112]$.

When $\beta$ is unknown, from (3.7) and (3.8), the Bayes point estimate of $\beta$ equals $\tilde{\beta}=0.6578$ while the two-sided $95 \%$ Bayes CI for $\beta$ is $[0.4607,0.8894]$. It is easy to obtain $z=54.73$ from (2.11). To investigate the posterior density of $\beta$, we first generate $m=50,000$ i.i.d. samples of $\beta$ according to (3.6). Figure 1(a) shows that the posterior density of $\beta$ is quite symmetric and should be well approximated by a normal distribution. We also generate $m=50,000$ i.i.d. posterior samples $\left\{X^{(\ell)}\right\}_{\ell=1}^{m}$ of $X$ based on (3.9). For each given pair $\left(\beta^{(\ell)}, X^{(\ell)}\right)$, we then calculate $\alpha^{(\ell)}=\tau\left(X^{(\ell)}\right)^{-1 / \beta^{(\ell)}}$ and $\left\{\alpha^{(\ell)}\right\}_{\ell=1}^{m}$ are the i.i.d. posterior samples of $\alpha$. Based on these i.i.d. posterior samples, the Bayes point estimator for $\alpha$ is given by $\tilde{\alpha}$ $=39.1501$ while the two-sided 95\% Bayes CI for $\alpha$ is $[2.5117,135.9328]$. Figure 1 (b) shows that the posterior density of $\alpha$ is obviously skewed.

(a) Posterior density for beta

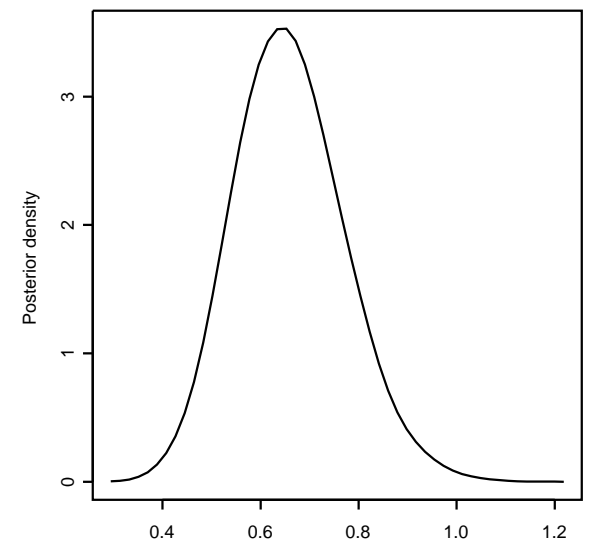

(b) Posterior density for alpha

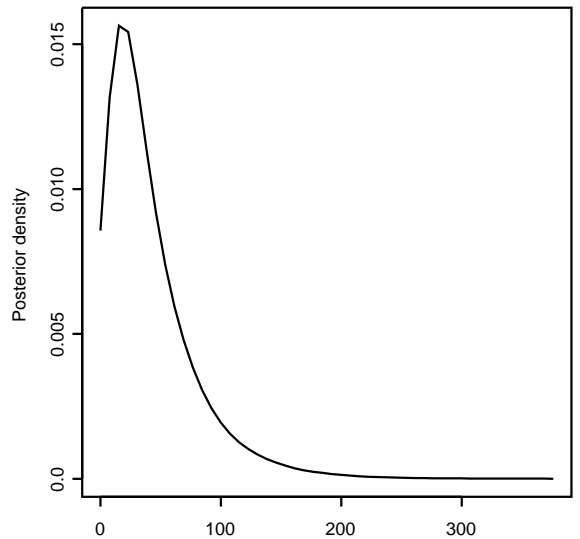

Figure 1: The posterior densities of $\beta$ and $\alpha$ generated by the conditional sampling method introduced in Section 3.1 with $m=50,000$ for the engine failure data.

Finally, from (3.12) and (3.13), we readily obtain the pseudo Bayes point estimate of $\lambda\left(x_{n}\right)$ as 0.00326 , and the two-sided $95 \%$ Bayes CI for $\lambda\left(x_{n}\right)$ as $[0.00195,0.00490]$.

\subsection{A repairable system failure data}


The following failure data from a prototype of a repairable system is given in ReliaSoft Corporation (2005). Briefly, a total of 12 failures for a system tested for $t=6500$ hours are given by: (80), (175), (265), (400), 590, 1100, 1650, 2010, 2400, 3380, 5100, 6400. ReliaSoft Corporation (2005) showed that the above life data followed a PLP. For illustrative purpose, we assume that the exact failure times for the first four failures are unknown. Since these data are time-truncated, we have $t=6500, n=12$ and $r=5$. The MLE of $\beta$ is $\hat{\beta}=(n-r+1) / z=$ 0.4389 .

We first consider the case that $\beta$ is known by letting $\beta=\hat{\beta}=0.4389$. From (3.3) and (3.4), the Bayes point estimator for $\alpha$ is $\tilde{\alpha}(\beta)=31.7042$ while the twosided $95 \%$ Bayes CI for $\alpha$ is $[7.3213,101.7389]$. From (3.15) and (3.16), the Bayes point estimator of $\lambda(t)$ is $\tilde{\lambda}(\beta)=0.00081$, and a two-sided $95 \%$ Bayes CI of $\lambda(t)$ is $[0.00042,0.00133]$.

When $\beta$ is unknown, we first generate $m=50,000$ i.i.d. posterior samples $\left\{\beta^{(\ell)}\right\}_{\ell=1}^{m}$ of $\beta$ according to (3.6). Thus, the Bayes point estimator for $\beta$ is $\tilde{\beta}=$ 0.3838 while the two-sided $95 \%$ Bayes CI for $\beta$ is [0.1542, 0.7151]. Figure 2(a) plots the posterior density of $\beta$. Next, for each given $\beta^{(\ell)}$, we generate $\lambda_{t}^{(\ell)}$ based on (3.20) for $\ell=1, \cdots, m$. Using these i.i.d. posterior samples of $\lambda(t)$, the Bayes point estimator for $\lambda(t)$ is $\tilde{\lambda}_{t}=0.000708$ and the two-sided $95 \%$ Bayes CI for $\lambda(t)$ is $[0.000226,0.00155]$. Figure 2(b) shows that the posterior density of $\lambda(t)$ is skewed.

(a) Posterior density for beta

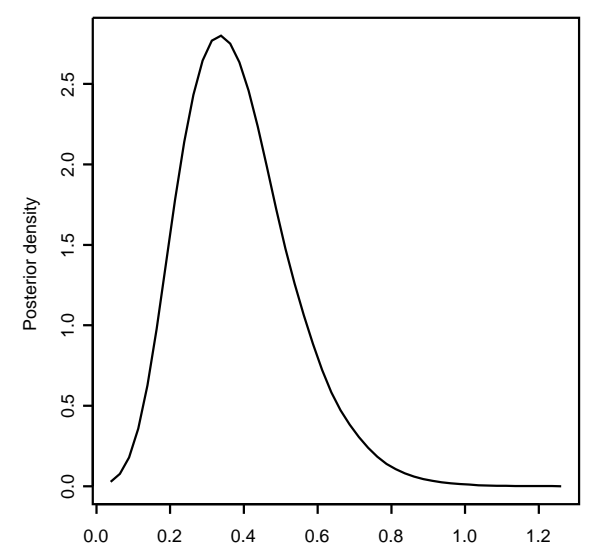

(b) Posterior density for lambda(t)

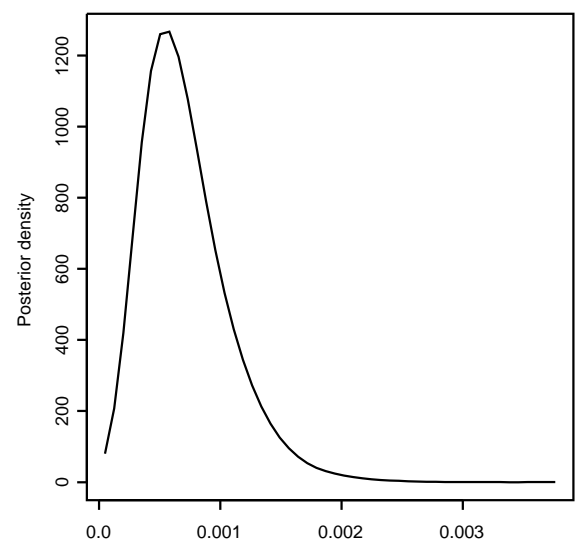

Figure 2: The posterior densities of $\beta$ and $\lambda(t)$ generated by the conditional sampling method introduced in Section 3.3 with $m=50,000$ for the repairable system failure data.

Now assume that we wish to obtain 0.95 Bayesian upper prediction limits on $x_{12+k}$ for $k=1$. When $\beta$ is known (say, $\beta=0.4389$ ), using (4.6), we have 
$x_{U, B}^{(\beta)}(12,1,0.95)=11479.9$. When $\beta$ is unknown, we obtained $y_{\gamma}=2.4925$ by solving (4.10). Thus, from (4.9), we get $x_{U, B}(12,1, r, 0.95)=14630.13$.

Suppose that we are interested in the probability $\gamma_{k}$ that at most $k$ failures will occur in the future time period $(\tau, T]=(6500,7500]$. (i) When $\beta$ is known (say, $\beta=0.4389$ ), using (4.16) and (4.17), we have $\gamma_{0}=0.4706, \gamma_{1}=0.8144$, $\gamma_{2}=0.9505, \gamma_{3}=0.9891, \gamma_{4}=0.9979, \gamma_{5}=0.99965$, and $\gamma_{6}=0.99995$. (ii) When $\beta$ is unknown, from (4.18) we obtain $\gamma_{0}=0.53246, \gamma_{1}=0.84436, \gamma_{2}=0.95668$, $\gamma_{3}=0.98912, \gamma_{4}=0.99743, \gamma_{5}=0.99941$ and $\gamma_{6}=0.99987$. Figure 3 shows the desired probabilities for known and unknown $\beta$.

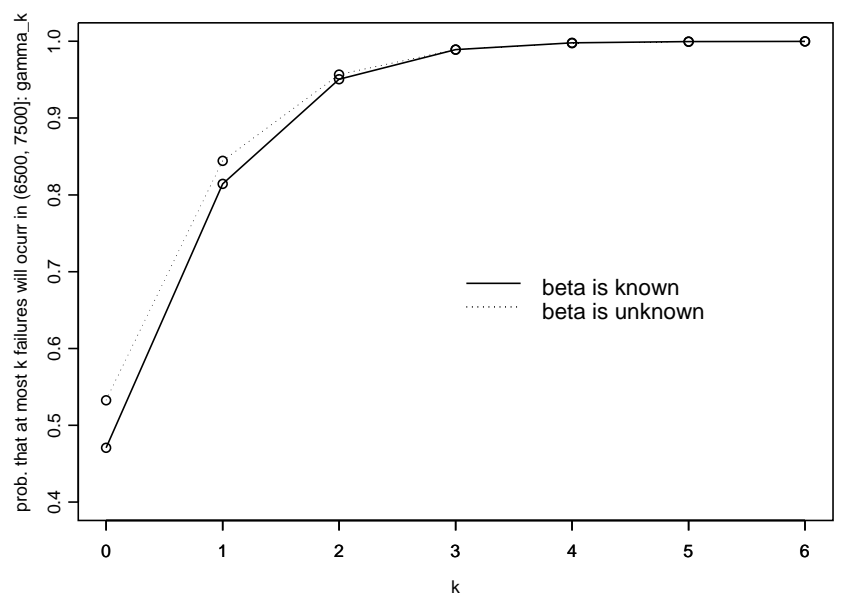

Figure 3: Comparison of the probabilities $\gamma_{k}$ that at most $k$ failures will occur in the time interval $(6500,7500]$ for known $\beta=0.4389$ and unknown $\beta$.

\section{Conclusion}

In this article, we consider Bayesian estimation and prediction methods for the PLP in the presence of left-truncated data. Bayesian point and credible interval estimates for the parameters of interest are derived. Bayesian prediction limits of future failure times are available for both failure- and time-truncated cases. We also show how five single-sample and three two-sample issues are addressed by the proposed Bayesian method.

It is interesting to note that (4.6) does not depend on $r$. In other words, when $\beta$ is known, the Bayes prediction limit of $x_{n+k}$ remains the same regardless of the absence of the first $(r-1)$ failure times (i.e., $\left.\left\{x_{i}\right\}_{i=1}^{r-1}\right)$. This result is not surprising because the conditional distribution of $x_{n+k}$ given $x_{1}, \cdots, x_{n+k-1}$ depends only on $x_{n+k-1}$ but not $x_{1}, \cdots, x_{n+k-2}$ (cf. (1.2)).

Furthermore, when $\beta$ is known, the Bayes estimates of $\alpha$ (see (3.3) and (3.4)), $\lambda(t)$ (see (3.15) and (3.16)), $\operatorname{Pr}\{N(\tau, T) \leq k\}$ (cf. (4.14)), $\operatorname{Pr}\left\{\lambda(T) \leq \lambda_{0}\right\}$ (cf. 
(4.24)), as well as the Bayes prediction limit of $y_{k}$ (cf. (5.3)), remain the same regardless of the absence of the first $(r-1)$ failure times. In other words, these posterior estimates and prediction depend on the data only through $n$ and $\tau$, and do not depend on $r$ and $x_{r}$.

In addition, we notice that the Bayesian UPL of $x_{n+k}$ under the noninformative prior (2.5) is identical to the classical UPL of $x_{n+k}$ by comparing (4.6) with (3.18) in Yu, Tian and Tang (2008). Most importantly, the prediction limit for $x_{n+k}$ is available only for the failure-truncated case under the classical approach while the prediction limits for $x_{n+k}$ are available for both the failure- and time-truncated cases under Bayesian approach.

By comparing (4.9) with (3.18) and (3.19) in Yu, Tian and Tang (2008), we find that the Bayesian UPL for $x_{n+k}$ under the joint noninformative prior (2.9) is identical to the classical UPL for $x_{n+k}$. Again, the Bayes prediction limits for $x_{n+k}$ are available for both failure- and time-truncated cases.

\section{Acknowledgments}

The research of JW Yu was fully supported by a grant (09C390) from Scientific Research Fund of Hunan Provincial Education Department.

\section{References}

Bain, L. J. (1978). Statistical Analysis of Reliability and Life-Testing Models. Marcel Dekker, New York.

Box, G. E. P. and Tiao, G. C. (1973). Bayesian Inference in Statistical Analysis. Reading, Addison-Wesley, Mass.

Calabria, R., Guida, M. and Pulcini, G. (1990). Bayes estimation of prediction intervals for a power law process. Communications in Statistics-Theory and Methods 19, 3023-3035.

Crow, L. H. (1974). Reliability analysis for complex repairable systems. In Reliability and Biometry - Statistical Analysis of Life Length (F. Proschan and R. J. Serfling, eds.), 379-410. SIAM, Philadelphia.

Crow, L. H. and Basu, A. P. (1988). Reliability growth estimation with missing data - II. IEEE Proceedings Annual Reliability and Maintainability Symposium, 248-253.

Crowder, M. J., Kimber, A. C., Smith, R. L. and Sweeting, T. J. (1991). Statistical Analysis of Reliability. Chapman and Hall, London. 
Duane, J. T. (1964). Learning curve approach to reliability monitoring. IEEE Transactions on Aerospace 2, 563-566.

Guida, M., Calabria, R. and Pulcini, G. (1989). Bayes inference for a nonhomogeneous Poisson process with power intensity law. IEEE Transactions on Reliability 38, 603-609.

Higgins, J. J. and Tsokos, C. P. (1981). A quasi-Bayes estimate of the failure intensity of a reliability growth model. IEEE Transactions on Reliability 30, 471-475.

ReliaSoft Corporation (2005). Reliability Growth and Repairable System Analysis Reference.

Rigdon, S. E. (2002). Properties of the Duane plot for repairable systems. Quality and Reliability Engineering International 18, 1-4.

Sen, A. (2002). Bayesian Estimation and Prediction of the Intensity of the Power Law Process. Journal of Statistical Computation and Simulation 72, 613-631.

Yu, J. W., Tian, G. L. and Tang, M. L. (2008). Statistical inference and prediction for the Weibull process with incomplete observations. Computational Statistics and Data Analysis 52, 1587-1603.

Zhou, Y. Q. and Weng, Z. Xi (1992). Reliability Growth. Science Press, Beijing.

Received February 20, 2010; accepted July 31, 2010.

Guo-Liang Tian

Department of Statistics and Actuarial Science

The University of Hong Kong

Pokfulam Road, Hong Kong, P. R. China

gltian@hku.hk

Man-Lai Tang

Department of Mathematics

Hong Kong Baptist University

Kowloon Tong, Hong Kong, P. R. China

mltang@math.hkbu.edu.hk

Jun-Wu Yu

School of Mathematics and Computational Science

Hunan University of Science and Technology

Xiangtan, Hunan 411201, P. R. China

jwyu.my265@yahoo.com.cn 